\title{
A Comparative Study of Feature Types for Age-Based Text Classification ${ }^{\star}$
}

\author{
Anna Glazkova1 ${ }^{1[0000-0001-8409-6457]}$ and Yury Egorovi[0000-0001-7670-5283] \\ and Maksim Glazkov2[0000-0002-4290-2059] \\ 1 University of Tyumen, ul. Volodarskogo 6, 625003 Tyumen, Russia \\ a.v.glazkova@utmn.ru, yurij.a.egorov@gmail.com \\ 2 "Organization of cognitive associative systems" LLC, ul. Gertsena 64, 625000 \\ Tyumen, Russia my.eye.off@gmail.com
}

\begin{abstract}
The ability to automatically determine the age audience of a novel provides many opportunities for the development of information retrieval tools. Firstly, developers of book recommendation systems and electronic libraries may be interested in filtering texts by the age of the most likely readers. Further, parents may want to select literature for children. Finally, it will be useful for writers and publishers to determine which features influence whether the texts are suitable for children. In this article, we compare the empirical effectiveness of various types of linguistic features for the task of age-based classification of fiction texts. For this purpose, we collected a text corpus of book previews labeled with one of two categories - children's or adult. We evaluated the following types of features: readability indices, sentiment, lexical, grammatical and general features, and publishing attributes. The results obtained show that the features describing the text at the document level can significantly increase the quality of machine learning models.
\end{abstract}

Keywords: Text classification · Fiction · Corpus · Age audience · Content rating - Text difficulty $\cdot$ RuBERT $\cdot$ Neural network · Natural language processing · Machine Learning.

\section{Introduction}

Nowadays, there are quite a lot of approaches to text classification according to document subjects, genre, author or according to other attributes. However, modern challenges in the field of natural language processing (NLP) and information retrieval (IR) increasingly require classification based on more complex characteristics. For example, it may be necessary to determine whether the text contains elements of propaganda or whether it has similar plot characteristics to other texts. One of such urgent and complex classification tasks is the division of literary texts into suitable for children and for adults. Age-based classification

\footnotetext{
* Supported by the grant of the President of the Russian Federation no. MK637.2020 .9
} 
tools could find wide practical application. For instance, they would be useful in the personal selection of fiction or in filtering content not intended for children.

Despite the fact that many scholars considered the issue of text difficulty estimation, formally, text difficulty does not indicate the age of the intended reader. The question of whether the features describing text difficulty are suitable for age-based classification needs to be investigated. In addition, the severity of the features depends on the text genre. It is necessary to find out how these or those features are presented in the literary text and whether they contain information about the age audience of the text.

In this paper, we systematically evaluated different feature types on age-based classification task. In addition to popular text difficulty features, we consider special publishing attributes intrinsic fiction books, such as age rating score and abstract features. We collected the corpus of Russian fiction texts and applied two commonly used machine learning models, these are random forest (RF) and linear support vector classifier (LSVC). For comparison, we evaluated a transformer model based on RuBERT and a convolutional neural network (CNN) trained on Word2Vec embeddings. Finally, we evaluated feedforward neural network (FNN) trained on RuBERT text embeddings and age rating scores.

The LSVC model using a combination of a baseline and publishing attributes showed the best result of $95.77 \%$ (F1-score). RuBERT achives $90.16 \%$. The FNN model combining RuBERT embeddings and age ratings showed $94.78 \%$. The results show that the features describing the text at the document level gives an advantage in case of long texts. Moreover, publishing attributes provide valuable information for the age-based classifier. We also found that some features used to determine text difficulty positively affect the quality of age-based classification.

The paper is organised as follows. In Section 2 we present a brief review of related works. Section 3 describes feature types evaluated in the paper. Section 4 contains the description of our dataset. Section 5 presents the structure of the models and the evaluation results. Finally, Section 6 is a conclusion.

\section{Related Works}

In the modern world, the constant growth of information resources gives rise to the need for filtering and ranking texts. One of the significant characteristics of a text is its complexity. The question of determining text difficulty naturally looks related to the task of age-based classification.

The task of estimating texts by complexity is not new. It appeared at the beginning of the last century in the context of evaluating the readability of educational texts. Further, during the XX century, researchers have proposed a number of tests to determine readability based on the quantitative characteristics of texts. Readability tests usually use quantitative text features, such as counting syllables, words, and sentences. There are several common readability texts for text difficulty estimation. For instance, these are: the Flesch-Kincaid readability test, the Coleman-Liau index, the automated readability index (ARI), the SMOG grade, the Dale-Chall formula 610. 
- The Flesch-Kincaid readability test is based on the idea that the shorter the sentences and words, the simpler the text. The specific mathematical formula is:

$$
R_{F}=206.835-1.015 \cdot A S L-84.6 \cdot A S W
$$

$A S L$ - average sentence length, $A S W$ - average number of syllables per word (i.e., the number of syllables divided by the number of words).

- The Coleman-Liau index uses letters instead of syllables. The formula takes into account the average number of letters per word and the average number of words per sentence.

$$
R_{C}=0.0588 \cdot L-0.296 \cdot S-15.8,
$$

where $L$ - average number of letters per 100 words, $S$ - average number of sentences per 100 words.

- The ARI formula takes into account the number of letters. In the past, this allowed the use of this index to measure the complexity of texts in real time in electric typewriters.

$$
R_{A}=4.71 \cdot \frac{\text { characters }}{\text { words }}+0.5 \cdot \frac{\text { words }}{\text { sentences }}-21.43,
$$

where characters - number of letters and numbers, words - number of words, sentences - number of sentences.

- The main idea of the SMOG grade is that the complexity of the text is most affected by complex words. Complex words are words with many syllables (more than 3). The more syllables the more complicated the word.

$$
R_{S}=1.043 \cdot \sqrt{\text { polysyllable } \cdot \frac{30}{\text { sentences }}}+3.1291,
$$

where polysyllable - number of polysyllable words, sentences - number of sentences.

- The Dale-Chall formula uses a count of "hard" words. These "hard" words are words that do not appear on a specially designed list of common words familiar to most 4th-grade students.

$$
R_{D}=0.1579 \cdot \frac{\text { difficult }}{\text { words }} \cdot 100+0.0496 \cdot \frac{\text { words }}{\text { sentences }}
$$

where difficult - number of difficult words, words - number of words, sentences - number of sentences.

In addition to the above, there are many other readability tests that are also actively used, e.g. the Fry Graph readability formula, the Spache index, the Linsear Write formula and others. The values obtained from readability tests are called readability indices. The Readability Index characterizes the difficulty of perceiving a text or the expected level of education that is required to understand it. 
The readability formulas listed above are metrics for English texts. At the same time, the quantitative characteristics of other languages can differ significantly. For instance, Russian sentences are on average shorter than English, and words are longer. Therefore, the readability formulas need to be processed for use in other languages. Up to now, several studies have suggested the adaptation of readability tests for Russian. For example, I. Oborneva 27 proposed the coefficients for the Flesch-Kincaid formula for Russian texts. The project 41 offers the adaptation of several readability formulas. M. Solnyshkina et al. presented a new approach to reading difficulty prediction in Russian texts [37/38.

Readability is however only one aspect of age-based classification. Scholars have proposed more complex techniques for text complexity estimation using features of different nature. Thus, Yu. Tomina 42 considered the lexical and syntactic features of the text complexity level. A. Laposhina et al. 22] evaluated a wide range of different types of features, such as readability, semantic, lexical, grammatical and others. M. Shafaei et al. 33. estimated age suitability rating of movie dialogs using genre and sentiment features. L. Flekova et al. [16] proposed an approach to describing the story complexity for literary text. Y. Bertills [4] wrote about the features of literary characters and named entities in books for children. Finally, in our previous research, we evaluated the informativeness of some quantitative and categorical features for age-based text classification [12. The modern methodology for text difficulty estimation is based in most cases on machine learning approaches. Thus, R. Balyan et al. 3] showed that applying machine learning methods increased accuracy by more than $10 \%$ as compared to classic readability metrics (e.g., Flesch-Kincaid formula). To date, a number of studies confirmed the effectiveness of various machine learning techniques for text difficulty estimation, such as support vector machine (SVM) [36 39, random forest [26], and neural networks [27/35].

Another aspect of assessing the age category of text readers is the safety of the information it contains. Currently, in many countries, publishers are required to label books (including fiction) and other informational sources 9/11/14]18]30 according to their age rating. For these purposes, there are special laws that rank information in terms of the potential harm it can bring. So, in Russia there is a Russian Age Rating System (RARS).

The RARS was introduced in 2012 when the Federal law of Russian Federation no. 436-FZ of 2010-12-23 "On Protection of Children from Information Harmful to Their Health and Development" was passed [15. The law prohibits the distribution of "harmful" information that depicts violence, unlawful activities, substance abuse, or self-harm. The RARS includes 5 categories, such as for children under the age of six $(0+)$, for children over the age of six $(6+)$, for children over the age of twelve (12+), for children over the age of sixteen (16+), and prohibited for children (18+). As a rule, an age rating is assigned to a book by editors or experts. As far as we know, there are currently no published research of how age rating correlates with other attributes of text, such as readability.

The reviewed studies and sources clearly indicate that age-based classification of fiction texts includes several aspects. First, the research topic is related to 
works on text difficulty evaluation. Text difficulty is characterized by different features, these are lexical, semantic, grammatical and other types. However, the measure of the difficulty of the text does not guarantee that this text is targeted to a particular age audience. It is required to evaluate the effectiveness of the existing text difficulty features for age-based classification. In addition, it would be interesting to evaluate the role of publishing attributes (for example, age rating labels) as classification features. Finally, the studies presented thus far provide evidence that machine learning approaches show the highest results in the task of estimating texts by difficulty. Based on this, it is reasonable to evaluate the text features for age-based classification using machine learning methods.

\section{Feature Types}

According to the related works, we consider the following types of classification features.

1. General features. This type includes features that reflect the quantitative characteristics of the text:

- the average and median length of words (avg_words_len, med_words_len);

- the average and median length of sentences (avg_sent_len, méd_sent_len), e.g. average or median number of symbols in each sentence;

- the average number of syllables (avg_count_syl);

- the percentage of long words with more than 4 syllables (many_syllables);

- the Type-Token Ration, TTR (ttr) [40. The main idea of the metric is that if the text is more complex, the author uses a more varied vocabulary so there's a larger number of unique words. So, the TTR's value is calculated as the number of unique words divided by the number of words. As a result, the higher the TTR, the higher the variety of words;

- the TTR for nouns $\left(t t r_{-} n\right)$, adjectives $\left(t t r_{-} a\right)$, and verbs $\left(t t r_{-} v\right)$. The values of TTR calculated separately for parts of speech;

- the NAV metric (nav). The NAV metric is a TTR-based ratio of (TTR A + TTR N)/TTR V proposed in 37.

2. Readability features. We used the readability formulas with the coefficients for the Russian language proposed by the project 41. In this study, we evaluated five types of readability indices using the following metrics: the Flesch-Kincaid readability test (index_fk); the Coleman-Liau index (index_cl); the ARI index (index_ari); the SMOG grade (index_SMOG); the Dale-Chall formula (index_dc).

3. Lexical features. In this category, we included features constructed by the evaluation of the text in accordance with frequency dictionaries. As frequency dictionaries, we used the lists of Russian frequency words presented in 25]34:

- the percentage of words included in the list of 5000 most frequent Russian words (5000_proc); 
- the average frequency of the words included in the 5000 most frequent words (5000_freq);

- the average frequency of words per 1 million occurrences (ipm, words_fr);

- the average frequency of nouns, verbs, adjectives, adverbs and proper names per 1 million occurrences $\left(s_{-} f r, v f r, a d j_{\_} f r, a d v_{-} f r, p r o p \_f r\right)$;

- the average number of topic segments of the corpus 3 where the word was encountered (out of 100 possible, words_r );

- the average number of the corresponding topic segments for nouns, verbs, adjectives, adverbs and proper names $\left(s_{-} r, v_{-} r, a d j_{-} r, a d v_{-} r, p r o p_{-} r\right)$;

- the average value of Juilland's usage coefficients (words_ $d$ ). This Juilland's usage coefficient measures the dispersion of the word's subfrequencies over $n$ equally-sized subcategories of the corpus [17;

- the average value of Juilland's usage coefficients for nouns, verbs, adjectives, adverbs and proper names $\left(s_{-} d, v_{-} d, a d j_{-} d, a d v_{-} d, p r o p_{-} d\right)$;

- the number of documents in the corpora in which a word occurs (averaged over the text, words_doc);

- the average number of documents in the corpora in which a word occurs (for nouns, verbs, adjectives, adverbs and proper names, $s_{-} f r, v \_f r, a d j f r$, $\left.a d v_{-} f r, p r o p \_f r\right)$.

4. Grammatical features. We evaluated the percentage of nouns, verbs, and adjectives (count_n, count_v, count_a).

5. Sentiment features. These features obtained with Russian Sentiment Lexicon 24. We separately evaluated the percentage of positive and negative words for each of the topic categories, these are opinion, feeling (private state), or fact (sentiment connotation) (neg_opinion, neg_feeling, neg_fact, pos_opinion, pos_feeling, pos_fact).

6. Publishing features. Here we have included features based on publishing attributes, i.e. on the book characteristics assigned by an editor or publisher, such as age rating according to the RARS (age_rating) and TF-IDF scores for book abstracts.

\section{Dataset}

For feature evaluation, we collected a dataset of fiction books published in Russian. Due to copyright restrictions, the full texts of the books are not publicly available. Therefore, we used a collection of previews presented in online libraries in the public domain. Typically, the preview is $5-10 \%$ of the total book volume.

The corpus consists of 5592 texts of children's and adult book previews. We have divided the texts into two parts. The first part included 4492 texts. It was used to train the models. The remaining 1000 texts were served as an independent text sample. The main characteristics of the data is presented in Table 1. Table 2 shows short text examples of adults and children's categories.

\footnotetext{
${ }^{3}$ The frequency dictionary was created on the basis of the modern subcorpus of the Main Corpus and the Oral Corpus of the Russian National Corpus (1950-2007) 32 with a total volume of 92 million tokens 25 .
} 
Table 1. Characteristics of the corpus.

\begin{tabular}{|l|l|l|l|l|}
\hline \multirow{2}{*}{ Characteristic } & \multicolumn{2}{|l|}{ Training sample } & \multicolumn{2}{|l|}{ Test sample } \\
\cline { 2 - 5 } & Children's & Adult & Children's & Adult \\
\hline Number of text & 2108 & 2384 & 500 & 500 \\
Avg number of symbols & 3134.38 & 3326.11 & 3048.69 & 3319.86 \\
Avg number of tokens & 488.55 & 499.52 & 479.3 & 498.16 \\
Avg number of sentences & 37.35 & 35.2 & 36.05 & 36.49 \\
\hline
\end{tabular}

Table 2: Example short fragments.

\begin{tabular}{|l|l|l|l|}
\hline Category & $\begin{array}{l}\text { Age } \\
\text { Rating }\end{array}$ & Genre & $\begin{array}{l}\text { Fragment } \\
\text { Adults }\end{array} 16+$ \\
$\begin{array}{l}\text { Modern } \\
\text { mance } \\
\text { novels }\end{array}$ & $\begin{array}{l}\text { A tall young man dressed in jeans, an inconspic- } \\
\text { a visor over his eyes, approached the entrance of } \\
\text { a seventeen-story apartment building and stood as } \\
\text { if waiting for someone, and when a mother with a } \\
\text { stroller appeared at the door, he quickly jumped } \\
\text { inside - he did not know the code. I walked up to } \\
\text { the fifth floor, putting on thin gloves on the go, } \\
\text { looked around, and then deftly opened the door of } \\
\text { one of the apartments. On the threshold he froze } \\
\text { and listened for a while, but it was quiet. The man } \\
\text { turned the baseball cap over the visor and began a } \\
\text { leisurely survey of the apartment, opening the doors } \\
\text { of the cupboards and looking into the drawers. The } \\
\text { first thing he did was to open the sliding wardrobe } \\
\text { door in the hallway, and oversized men's slippers, } \\
\text { an empty box, and a bright purple scarf fell out } \\
\text { onto the floor. The man grimaced and shoved ev- } \\
\text { erything back, muttering, I thought so." In a large } \\
\text { room that served as a bedroom, he lingered a lit- } \\
\text { tle longer and grunted ironically at the sight of a } \\
\text { luxurious couch with a carved back. 4 }\end{array}$ \\
\hline
\end{tabular}

\footnotetext{
${ }^{4}$ Fragment from the book "Men We Choose" by Evgeniya Perova (translated from Russian).
} 


\begin{tabular}{|c|c|c|c|}
\hline Adults & $12+$ & $\begin{array}{l}\text { Histori- } \\
\text { cal } \\
\text { adven- } \\
\text { tures }\end{array}$ & $\begin{array}{l}\text { What a wonderful autumn it was in Southern } \\
\text { Poland that year! Almost without rain and cold } \\
\text { winds, tenderly warm, quiet, crimson-gold. Fabu- } \\
\text { lous autumn - in such an autumn it is good, having } \\
\text { climbed into the spurs of the Beskydy, from dawn to } \\
\text { noon to wander along the slopes of hills overgrown } \\
\text { with beech and hazel, and to your fill, drunk to } \\
\text { breathe in the cool and crystal clear mountain air. } \\
\text { And then, on the cozy terrace of a small mountain } \\
\text { tavern, eat a good portion of hot, fiery-spicy bigos } \\
\text { with pork legs, washed down with icy "okocim". } \\
\text { And in the evening, having walked up to aching } \\
\text { knees, kindle a fire on a platform open to all the } \\
\text { winds above a shallow ravine, and, sitting on un- } \\
\text { bound logs, look at the stars that suddenly poured } \\
\text { out in incredible numbers overhead. And, peering } \\
\text { to the north, in the transparent thickening blue of } \\
\text { the air, distinguish the lights of distant Krakow or } \\
\text { Nova Huta, or maybe Bochnia or Wieliczka, who } \\
\text { knows? } 5\end{array}$ \\
\hline \begin{tabular}{|l|} 
Children's \\
\end{tabular} & $6+$ & $\begin{array}{l}\text { Child- } \\
\text { ren's } \\
\text { adven- } \\
\text { tures }\end{array}$ & $\begin{array}{l}\text { In a big, big city, where there are many, many } \\
\text { houses, many, many cars and even more people, and } \\
\text { the crows cannot be counted at all, there lived a } \\
\text { ginger cat on a short street consisting of only two } \\
\text { courtyards. His name was Ostrich. } 6\end{array}$ \\
\hline \begin{tabular}{|l|} 
Children's \\
\end{tabular} & $12+$ & $\begin{array}{l}\text { Child- } \\
\text { ren's } \\
\text { fan- } \\
\text { tastic } \\
\text { tales }\end{array}$ & $\begin{array}{l}\text { The hands of the clock were approaching half past } \\
\text { seven, but the setting sun, reluctantly sliding be- } \\
\text { hind the houses, continued to burn the city with } \\
\text { rays, and the approaching twilight did not promise } \\
\text { the long-awaited coolness. } \\
\text { Friday night was hot and stuffy, and the city roofs } \\
\text { were so hot during the day that no sane cat would } \\
\text { dare to run over them without burning their paws. } \\
\text { August was coming to an end, and the sun knew } \\
\text { that it was the most important thing in the city, } \\
\text { so from the very morning it climbed everywhere, } \\
\text { trying to melt the asphalt on the streets, drying the } \\
\text { grass on the lawns and sneaking into the apartments } \\
\text { to flood them with heat and stuffiness. } 7\end{array}$ \\
\hline
\end{tabular}

\footnotetext{
5 "Sold Poland" by Alexander Usovsky (translated from Russian).

6 "Greetings from cutlets" by Evgenia Malinkina (translated from Russian).

7 "Vlad and the Secret Ghost" by Sasha Gotti (translated from Russian).
} 
Table 3 shows the most informative quantitative features with their means and standard deviation values. The informativeness is measured using the method of cumulative frequencies 1144. The main idea of this method consists in dividing the range of feature values for each class into $n$ intervals. The cumulative frequency of characteristic values is calculated for each interval. The informativeness indicator is calculated as the maximum absolute value of the difference in the accumulated frequencies for the corresponding intervals in the classes.

Table 3. Top-10 of the most informative quantitative features (according to the method of cumulative frequencies).

\begin{tabular}{|l|l|l|l|l|}
\hline Feature & Mean (adult) & std (adult) & Mean (children's) & std (children's) \\
\hline avg_sent_len & 105.65 & 54.51 & 88.69 & 30.64 \\
med_sent_len & 97.9 & 59.06 & 79.69 & 34 \\
index_dc & 7.85 & 2.64 & 6.36 & 2.09 \\
adj_doc & 3484.28 & 1074.03 & 3669.48 & 1173.78 \\
index_ari & 9.29 & 3.53 & 7.4 & 2.93 \\
adj_fr & 135.3 & 53.56 & 146.96 & 60.39 \\
s_doc & 3705.73 & 838.45 & 3370.52 & 766.39 \\
index_fk & 9.21 & 3.66 & 7.36 & 2.95 \\
v_doc & 6255.7 & 2243.76 & 6481.53 & 1937.8 \\
adv_fr & 239.17 & 84.26 & 277.43 & 90.93 \\
\hline
\end{tabular}

Figure 1 presents the distribution of the age rating labels (age rating is a categorical feature) in the classes of the training data. It is interesting to note that some of the books from the children's class are labelled with the 18+ age category. Notable examples of this type of books are love stories for teens.

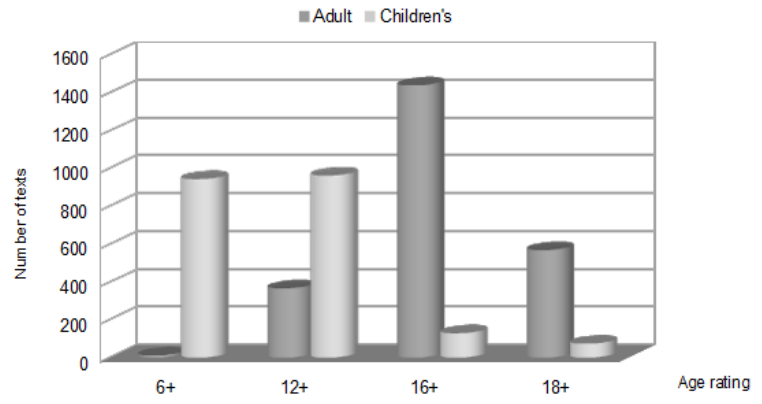

Fig. 1. The distribution of age rating categories. 


\section{Experiments}

This section describes our feature evaluation experiments. We built two types of baseline models and sequentially enriched them with different types of features. Further, we compared the results obtained with our models with the results of CNN and RuBERT. Our dataset and models are available at [5].

\subsection{Baselines}

We built two classifiers for model evaluation. The first one was a Random Forest Classifier trained on bootstrap samples. The number of trees in the forest was equal to 100 and the Gini impurity was implemented to measure the quality of a split. The second model was a Linear Support Vector Classifier with the "12" penalty and the squared hinge loss function. Both models were implemented using Scikit-learn 29] and Python 3.6.

\subsection{Preprocessing}

To preprocess our data, we used min-max normalization. Moreover, it is obvious that some of the features are correlated. For instance, most readability indices show a cross-correlation greater than 0.8. Another example of correlated feature pairs is average and median length of sentences or TTR values for all words and for particular parts of speech (Figure 2). To reduce the influence of feature correlation on the LSVC model, we applied linear dimensionality reduction using Singular Value Decomposition of the data with the minimum number of principal components such that $95 \%$ of the variance is retained.

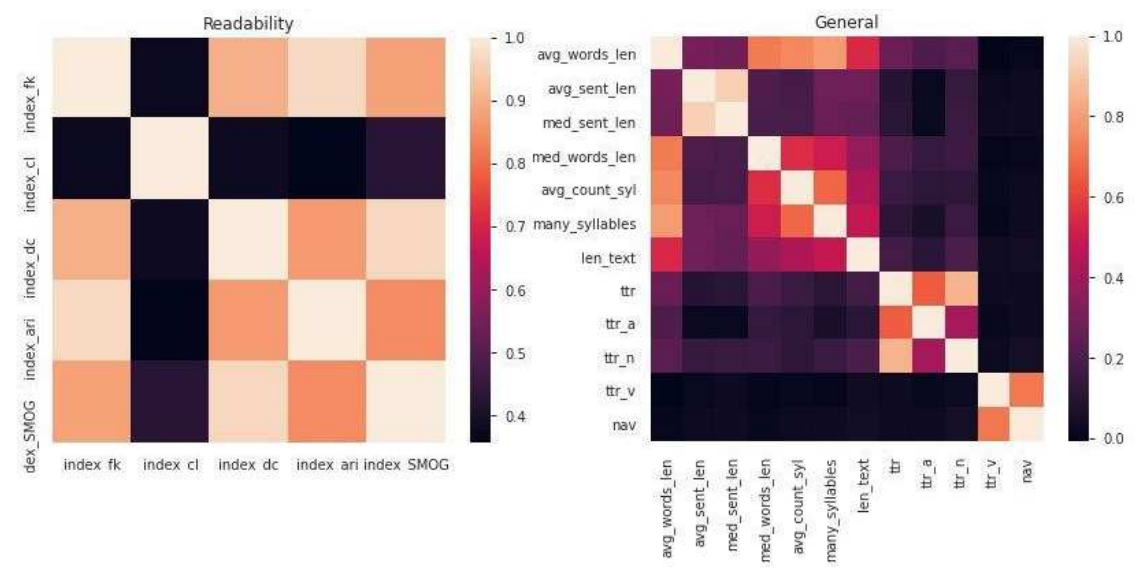

Fig. 2. Correlation matrices for readability and general features. 


\subsection{Experiments and Results}

We used models trained of TF-IDF vectors as baselines. Further, we systematically evaluated each type of features.

To begin this process, we connected the TF-IDF vector of the text with the corresponding vector of features of a certain type. Book previews are rather long texts. Since the RuBERT model that participated in the comparison can only process a sequence of limited length, we used the same fragments of 256 tokens to train both neural networks and to construct TF-IDF vector: 8 . TF-IDF vectors were built over the top 2000 words ordered by term frequency across the corpus. At the same time, the values of additional features were calculated for the full preview texts.

To build TF-IDF vectors and CNN, the texts were pre-processed. The preprocessing included the following steps: special character removal, lowercase translation, lemmatization, stop-word removal. Text preprocessing was implemented using NLTK 23 and Pymorphy2 [19].

Table 4 shows the results obtained for each type of features (e.g. RF baseline + readability, LSVC baseline + readability) and the results of the models trained only on additional features without TF-IDF vectors (e.g. RF (readability), LSVC (readability)). For publishing attributes, we evaluated two separate types of models. The first type used book abstracts as supplementary information. In other words, we added the texts of abstract to the book preview and built new TF-IDF vectors. The second type used baseline TF-IDF vectors with an additional feature of age rating. Finally, we evaluated three types of combined models, such as using all considered features, only all additional features, and all features with the exception of editorial attributes. The results obtained were compared with the results of three neural models:

- RuBERT [20, based on BERT architecture [8]. BERT showed state-of-theart results on a wide range of NLP tasks. RuBERT was trained on the Russian part of Wikipedia and news data. The model was implemented using PyTorch 28] and Transformers [43] libraries, it was trained for 3 epoches;

- FNN trained on fine-tuned RuBERT [20] text embeddings obtained with PyTorch library 28]. Text embeddings were calculated by averaging the token vectors of the last hidden state. Age rating was presented as a onehot numeric array which is the most widely used coding scheme [31. FNN consisted of three layers including an input layer, a 1024 hidden layer with hyperbolic tangent activation function, and an output layer with softmax activation function. We also used Adam as an optimizer and binary crossentropy loss. The FNN model was implemented using Keras 13 library;

- CNN trained on Word2Vec embeddings 21. CNN consisted of four building units including three convolutional units $(\mathrm{CU})$ and a fully connected unit. Each CU contained the following sequence of layers $C-B N-C-B N-P$ where $C$ is a convolutional layer (CL), $B N$ is a batch normalization layer,

8 The maximum sequence length for BERT is 512 tokens. However, due to the rather large volume of the corpus, we were also limited in computational resources. 
Table 4. Age-based classification results (\%).

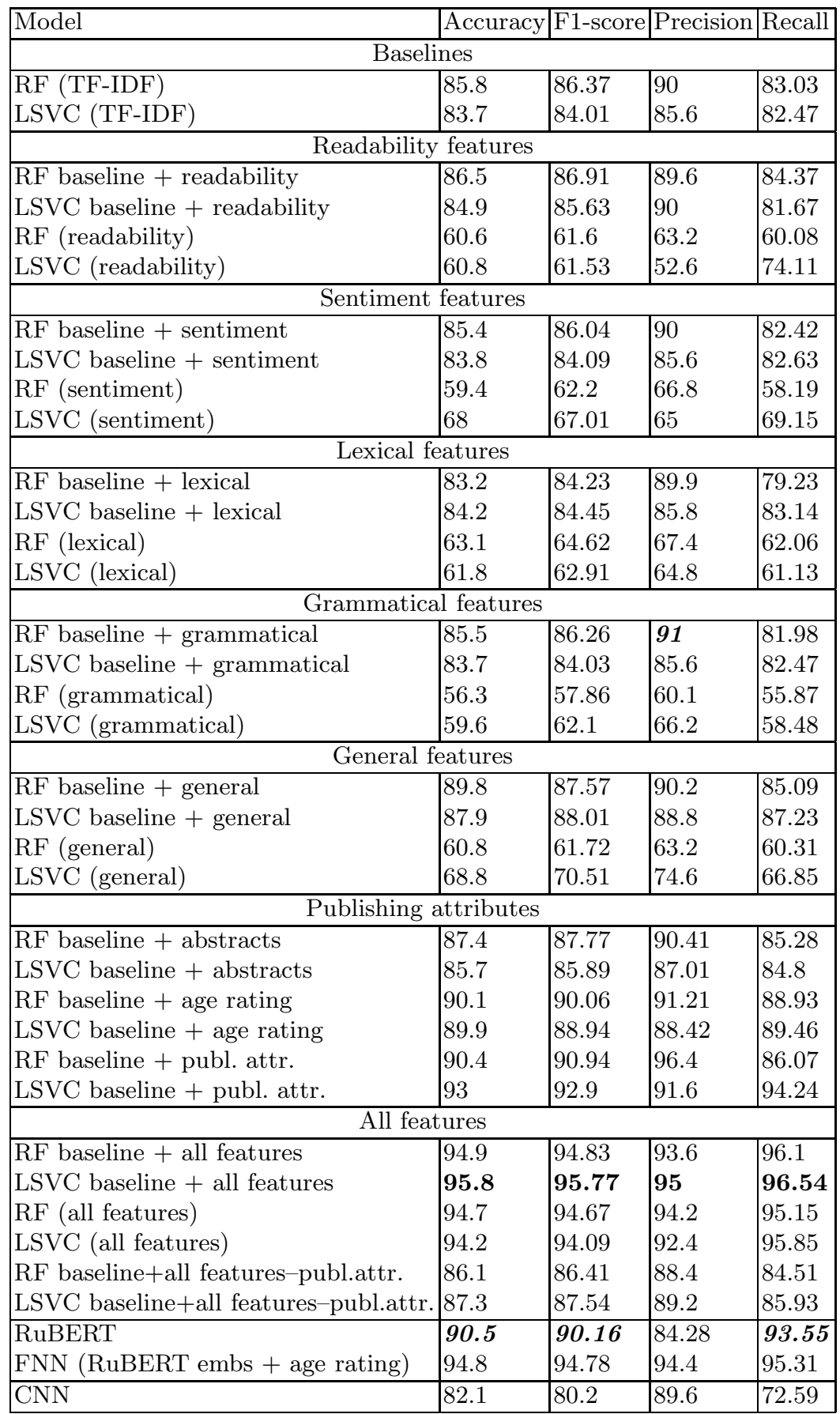


$P$ is a pooling layer. After every CL the LeakyRelu activation function was applied. At the first $\mathrm{CU}$ we used 512 filters $7 \times 7$. At the second CL we applied 1024 filters $5 \times 5$. As a pooling strategy at each layer we used max polling with a kernel $2 \times 2$. The fully connected layer consisted of the following sequence of layers $F L_{1}-B N-F N_{2}$ where $F L_{1}$ is a hidden layer with 32 neurons, $F L_{2}$ is an output layer. We applied ReLU as an activation function and used stochastic gradient descent with Nesterov momentum and learning rate equal to $5 \times 10^{-2}$ as optimization parameters. The model was implemented with PyTorch [28.

The results show that additional features in most cases improve the quality of baselines. According to F1-score, this concerns readability features, general features and publishing attributes. We assume that the advantage of these features is that they describe the text at the document level and allow the model to evaluate the whole text, and not just a fragment. It also can be seen that the using of abstracts and the age rating feature significantly improves the quality of the classification. The best results was obtained by the LSVC model using all considered features (95.8\% of accuracy, $95.77 \%$ of F1-score, $95 \%$ of precision, and $96.54 \%$ of recall). These values are shown in bold in Table 3. Among the models that did not use publishing attributes, the best results were shown by RuBERT (90.5\% of accuracy, $90.16 \%$ of F1-score, and $93.55 \%$ of recall) and the LSVC baseline with grammatical features ( $91 \%$ of precision).

\section{Conclusion}

The purpose of the current study was to evaluate different types of features for the task of age-based text classification. The results of this investigation show that features used in text difficulty evaluation can improve the quality of age-based classification. In addition, in this study, we considered publishing attributes (such as book abstracts and age ratings) as classification features. The results showed that the use of these attributes in digital libraries and recommendation systems could significantly improve the quality of machine learning approaches. Our further research will focus on studying other types of features, such as named entity analysis or plot and character features.

\section{References}

1. Aivazyan, S.A., Bukhshtaber, V.M., Enyukov, I.S. et al.: Applied Statistics: Classification and Dimension Reduction: A Handbook. Finansy i statistika, Moscow (1989).

2. Azpiazu, I.M., Pera, M.S.: Multiattentive recurrent neural network architecture for multilingual readability assessment. Trans. of the Association for Comp. Ling. 7, 421-436. https://doi.org//10.1162/tacl_a_00278

3. Balyan, R., McCarthy. K.S., McNamara, D.S.: Applying Natural Language Processing and Hierarchical Machine Learning Approaches to Text Difficulty Classification. Int. J. of Art. Intelligence in Education, 1-34 (2020). https://doi.org/10.1007/s40593-020-00201-7 
4. Bertills, Y.: Beyond identification : proper names in children's literature. Abo Akademi University Press, Turku (2003).

5. Corpus and Baselines for Age-Based Text Classification, https://github.com/oldaandozerskaya/age_based_classification. Last accessed 24 Sep 2020.

6. Crossley, S.A., Skalicky, S., Dascalu, M. et al.: Predicting text comprehension, processing, and familiarity in adult readers: New approaches to readability formulas. Discourse Processes 54, 5-6 (2017). https://doi.org/10.1080/0163853x.2017.1296264

7. Cuzzocrea, A., Bosco, G. L., Pilato, G. et al.: Multi-class text complexity evaluation via deep neural networks. LNCS 11872, 313-322 (2019). https://doi.org/0.1007/978-3-030-33617-2_32

8. Devlin, J., Chang, M.W., Lee, K. et al.: Bert: Pre-training of deep bidirectional transformers for language understanding. arXiv preprint arXiv:1810.04805 (2018).

9. Dogruel, L., Joeckel, S.: Video game rating systems in the US and Europe: Comparing their outcomes. International Communication Gazette 757, 672-692 (2013).

10. Didegah, F., Thelwall, M.: Which factors help authors produce the highest impact research? Collaboration, journal and document properties. J. of informetrics $7(4)$, 861-873 (2013). https://doi.org/10.1016/j.joi.2013.08.006

11. Grealy, L., Driscoll, C., Cather, K.: A history of age-based film classification in Japan. Japan Forum (2020). https://doi.org/10.1080/09555803.2020.1778058

12. Glazkova, A.: An Approach to Text Classification based on Age Groups of Addressees. SPIIRAS Proceedings 52(3), 51-69 (2017). https://doi.org/10.15622/sp.52.3

13. Gulli, A., Pal, S. Deep learning with Keras. Packt Publishing Ltd (2017).

14. Kim, S.W. et al. A Global Comparative Study on the Game Rating System. Journal of Digital Convergence 1712, 91-108 (2019).

15. Federal Law of Dec. 29, 2010 N 436-FZ "On the Protection of Children from Information Harmful to Their Health and Development", http://www.consultant.ru/document/cons_doc_LAW_108808/ Last accessed 23 Jul 2020.

16. Flekova, L., Stoffel, F., Gurevych, I. et al.: Content-based Analysis and Visualization of Story Complexity. In: Visualisierung sprachlicher Daten, pp. 185-223. Heidelberg: Heid. Univ. Publishing (2018).

17. Juilland, A.G., Brodin, D.R., Davidovitch, C.: Frequency dictionary of French words. Hague, Paris (1971).

18. Hamid, R.S., Shiratuddin, N.: Age Classification of the Existing Digital Game Content Rating System Across the World: A Comparative Analysis. In Proceedings of KMICe, pp. 218-222 (2018).

19. Korobov, M.: Morphological analyzer and generator for Russian and Ukrainian languages. In: International Conference on Analysis of Images, Social Networks and Texts, pp. 320-332. Springer, Cham (2015). https://doi.org/10.1007/978-3-319-26123-2_31

20. Kuratov, Y., Arkhipov, M.: Adaptation of deep bidirectional multilingual transformers for Russian language, arXiv preprint arXiv:1905.07213. 2019.

21. Kutuzov, A., Kuzmenko, E.: WebVectors: A Toolkit for Building Web Interfaces for Vector Semantic Models. CCIS 661, 155-161 (2017). https://doi.org/10.1007/978-3-319-52920-2_15

22. Laposhina, A.N., Veselovskaya, T.S., Lebedeva, M.U. et al.: Automated text readability assessment for Russian second language learners. In: Komp. Lingv. i Intel. Tehn., pp. 396-406 (2018). 
23. Loper, E., Bird, S.: NLTK: the natural language toolkit, arXiv preprint cs/0205028 (2002).

24. Loukachevitch, N., Levchik, A.: Creating a General Russian Sentiment Lexicon. In: Proc. of LREC-2016, pp. 1171-1176 (2016).

25. Lyashevskaya, O. N., Sharov S. A.: Frequency Dictionary of the Modern Russian Language (based on the materials of the National Corps of the Russian Language). Azbukovnik, Moscow (2009).

26. Mukherjee, P., Leroy, G., Kauchak D.: Using Lexical Chains to Identify Text Difficulty: A Corpus Statistics and Classification Study. J. of Biomed. and Health Informatics 23(5), 2164-2173 (2019). https://doi.org/10.1109/jbhi.2018.2885465

27. Oborneva, I. V.: Automated estimation of complexity of educational texts on the basis of statistical parameters. Pedagogy Cand. Diss. Moscow (2006).

28. Paszke, A., Gross, S., Massa, F. et al.: Pytorch: An imperative style, highperformance deep learning library. In: Adv. in neural information processing systems, pp. 8026-8037 (2019).

29. Pedregosa, F., Varoquaux, G., Gramfort, A. et al.: Scikit-learn: Machine learning in Python. The J. of Machine Learning research 12, 2825-2830 (2011).

30. Piasecki, S., Malekpour, S.: Morality and religion as factors in age rating computer and video games: ESRA, the Iranian games age rating system. Online-Heidelberg Journal of Religions on the Internet, 11.

31. Potdar, K., Pardawala, T. S., Pai, C. D.: A comparative study of categorical variable encoding techniques for neural network classifiers. International journal of computer applications 1754, 7-9 (2017). https://doi.org/10.5120/ijca2017915495

32. Russian National Corpus, https://ruscorpora.ru/new/en/index.html Last accessed 23 Jul 2020.

33. Shafaei, M., Samghabadi, N.S., Kar, S., Solorio, T.: Age Suitability Rating: Predicting the MPAA Rating Based on Movie Dialogues. In: Proceedings of The 12th Language Resources and Evaluation Conference, pp. 1327-1335 (2020).

34. Sharoff, S.: Meaning as use: exploitation of aligned corpora for the contrastive study of lexical semantics. In: Proc. of LREC02, pp. 447-452. Las Palmas, Spain (2002).

35. Schicchi, D., Pilato, G., Bosco, G.L.: Deep Neural Attention-Based Model for the Evaluation of Italian Sentences Complexity. In: 2020 IEEE 14th ICSC, pp. 253-256. https://doi.org/10.1109/icsc.2020.00053

36. Schwarm, S. E., Ostendorf, M. Reading level assessment using support vector machines and statistical language models. In: Proc. of ACL'05, pp. 523-530 (2005). https://doi.org/10.3115/1219840.1219905

37. Solnyshkina, M., Ivanov, V., Solovyev, V.: Readability Formula for Russian Texts: A Modified Version. In: Proc. of MICAI 2018, part II, pp. 142-145. Springer, Cham (2018). https://doi.org/10.1007/978-3-030-04497-8_11

38. Solovyev, V., Solnyshkina, M., Ivanov, V. et al.: Prediction of reading difficulty in Russian academic texts. J. of Int. \& Fuzzy Systems 36(5), 4553-4563 (2019). https://doi.org/10.3233/jifs-179007

39. Sung, Y.T., Chen, J.L., Cha, J.H. et al.: Constructing and validating readability models: the method of integrating multilevel linguistic features with machine learning. Behavior research methods 47(2), 340-354 (2015). https://doi.org/10.3758/s13428-014-0459-x

40. Templin, M. C.: Certain language skills in children; their development and interrelationships. Univ. of Minnesota Press, Minneapolis (1957).

41. Text readability rating, http://readability.io/ Last accessed 23 Jul 2020. 
42. Tomina, Yu. A.: Objective assessment of the language difficulty of texts (description, narration, reasoning, proof). Pedagogy Cand. Diss. Moscow (1985).

43. Wolf, T., Debut, L., Sanh, V. et al.: HuggingFace's Transformers: State-of-the-art Natural Language Processing. ArXiv, arXiv-1910 (2019).

44. Zagoruiko, N.G.: Applied methods of data and knowledge analysis. Izd-vo IM SO RAN, Novosibirsk (1999). 